INPLASY

PROTOCOL

To cite: Li et al. Acute effects of dynamic stretching, static stretching on vertical jump and sprint performance -meta analysis. Inplasy protocol 202160002. doi:

10.37766/inplasy2021.6.0002

Received: 03 June 2021

Published: 03 June 2021

Corresponding author: Qiang Li

lq18552125291@126.com

Author Affiliation: Graduate student of Nanjing Sport Institute

Support: School research fund project.

Review Stage at time of this submission: Preliminary searches.

Conflicts of interest:

None declared.

\section{Acute effects of dynamic stretching, static stretching on vertical jump and sprint performance - meta analysis}

Review question / Objective: Acute effects of dynamic stretching, static stretching on vertical jump and sprint performance.

Condition being studied: Pre-exercise warm-up routines involving stretching exercises performed after light aerobic activity have been advocated to reduce injury and enhance athletic performance. Recently, however, it has been shown that static stretching (SS) before athletic performance may actually decrease performance in a number of explosive activities. This has led researchers to question the use of SS performed before athletic events requiring high power outputs. However, some researchers conclude that a dynamic stretching (DS) routine may be a safer and more efficient alternative to SS before athletic performance and should be implemented into warm-up routines. But the inconsistencies in the extant literature.

INPLASY registration number: This protocol was registered with the International Platform of Registered Systematic Review and Meta-Analysis Protocols (INPLASY) on 03 June 2021 and was last updated on 03 June 2021 (registration number INPLASY202160002).

\section{INTRODUCTION}

Review question / Objective: Acute effects of dynamic stretching, static stretching on vertical jump and sprint performance.

Condition being studied: Pre-exercise warm-up routines involving stretching exercises performed after light aerobic activity have been advocated to reduce injury and enhance athletic performance . Recently, however, it has been shown that static stretching (SS) before athletic performance may actually decrease performance in a number of explosive activities. This has led researchers to question the use of SS performed before athletic events requiring high power 
outputs. However, some researchers conclude that a dynamic stretching (DS) routine may be a safer and more efficient alternative to SS before athletic performance and should be implemented into warm-up routines. But the inconsistencies in the extant literature.

\section{METHODS}

Participant or population: In a randomized controlled trial, the participants are healthy adults, aged between 18-40, with or without training experience, the warm-up method is dynamic stretching or static stretching, and no sports supplements (glucose, creatine) are taken during the test. And sports drinks, etc.), test collection indicators include vertical jump or sprint test.

\section{Intervention: Dynamic stretch.}

\section{Comparator: Static stretch.}

Study designs to be included: The literature screening process is completed by two independent researchers. By reading the title and abstract, research literature that does not meet the inclusion criteria of this study is eliminated, and the full text of the included literature is further read to eliminate the inconsistency of the physiological index method or time in the evaluation of this study. Related research literature. And then determine the final inclusion in the literature. Extracting features of included literature in Excel includes: first author and publication time, research object, age, sample size, intervention.

Eligibility criteria: Randomized controlled trial, The participants are healthy adults, aged between 18-40, with or without training experience, The warm-up method is dynamic stretching or static stretching, and no sports supplements (glucose, creatine) are taken during the test. And sports drinks, etc.), The test collection indicators include vertical jump or sprint test.
Information sources: Google Scholar, wOS, PubMed.

Main outcome(s): Acute effects of dynamic stretching, static stretching on vertical jump and sprint performance. The main outcome indicators include explosive power (vertical jump), sprint indicators (10meter sprint, 20-meter sprint, 30-meter sprint).

Quality assessment / Risk of bias analysis: The methodological risk bias evaluation of the literature refers to the Cochrane system evaluation standard to evaluate the quality of the included literature. The method includes 6 evaluation items: (1) random allocation method; (2) allocation plan hiding; (3) research object and treatment plan Implementers and measurers of research results use blind methods; (4) Data integrity of results; (5) Selective reporting of research results; (6) Other sources of bias. The three symbols of "green dot" (with clear description), "red dot" (without clear description), and "yellow dot" (unknown or insufficient description) are used to evaluate each included literature, as shown in Figure 2 and Figure 3. The modified version of the Jadad scale was used to evaluate the quality of 13 included literatures. The evaluation criteria were: 1-3 as low quality and 4-7 as high quality.

Strategy of data synthesis: RevMan5.3 software was used for statistical analysis. All outcome indicators in this study are continuous data. If the outcome indicators use different scales and units, the Standard Mean Difference (SMD) is used as the effect indicator. If the outcome indicators use the same scale and unit, The weighted mean difference (WMD) is used as the effect indicator. Each effect size was expressed with $95 \% \mathrm{Cl}$, and the difference was statistically significant with p0.1, $12<50 \%$, it means that the heterogeneity between studies is small, and the fixedeffects model is used for Meta analysis; when p50\%, It means that the heterogeneity between the studies is large, and the random effects model is used for 
Meta analysis, and the source of heterogeneity is found through subgroup analysis or sensitivity analysis.

Subgroup analysis: A subgroup analysis is conducted based on the participants' sports experience. Training experience includes: elementary (without any training experience or physical training for less than 1 year), intermediate (with 1-2 years of physical training experience), and advanced (with more than 2 years of physical training experience).

Sensitivity analysis: Re-analyze the metaanalysis through sensitivity analysis of the 13 included articles, including changing different effect models and eliminating individual studies one by one, etc.

Country(ies) involved: China.

Keywords: dynamic stretch, static stretch, jump, sprint.

Contributions of each author:

Author 1 - Qiang Li - The author contributed to the development of the selection criteria, and the risk of bias assessment and drafted the manuscript.

Email: lq18552125291@126.com

Author 2 - Qiang Ye - drafted the manuscript.

Email: yeqiang@nsi.edu.cn

Author 3 - Tianya Cao - The author provided statistical expertise.

Email: 382194089@qq.com 